University of Nebraska - Lincoln

DigitalCommons@University of Nebraska - Lincoln

Educational Psychology Papers and

Publications

Educational Psychology, Department of

April 1999

\title{
A Cueing Procedure to Control Impulsivity in Children with Attention Deficit Hyperactivity Disorder
}

Heidi D. Posavac

University of Rochester

Susan M. Sheridan

University of Nebraska-Lincoln, ssheridan2@unl.edu

Steven S. Posavac

University of Rochester

Follow this and additional works at: https://digitalcommons.unl.edu/edpsychpapers

Part of the Educational Psychology Commons

Posavac, Heidi D.; Sheridan, Susan M.; and Posavac, Steven S., "A Cueing Procedure to Control Impulsivity in Children with Attention Deficit Hyperactivity Disorder" (1999). Educational Psychology Papers and Publications. 33.

https://digitalcommons.unl.edu/edpsychpapers/33

This Article is brought to you for free and open access by the Educational Psychology, Department of at DigitalCommons@University of Nebraska - Lincoln. It has been accepted for inclusion in Educational Psychology Papers and Publications by an authorized administrator of DigitalCommons@University of Nebraska - Lincoln. 
Published in Behavior Modification 23:2 (April 1999), pp. 234-253. Copyright (C) 1999 Sage Publications, Inc. Used by permission. DOI: 10.1177/0145445599232003 Online at: http://bmo.sagepub.com/cgi/content/abstract/23/2/234

Authors'Note: We are grateful to Dr. Steven Szykula for allowing us the use of his facilities. We also thank Cathy Choi-Pearson and Tara Tent for implementing the cueing procedure and Amber ReMillard for her assistance in data collection.

Corresponding author-Susan M. Sheridan, University of Nebraska-Lincoln, Educational Psychology, 220 Bancroft Hall, Lincoln, NE 68588-0345.

\section{A Cueing Procedure to Control Impulsivity in Children with Attention Deficit Hyperactivity Disorder}

\author{
Heidi D. Posavac, University of Rochester \\ Susan M. Sheridan, University of Nebraska-Lincoln \\ Steven S. Posavac, University of Rochester
}

\begin{abstract}
This study tested the efficacy of a cueing procedure for improving the impulse regulation of four boys with Attention Deficit Hyperactivity Disorder (ADHD) during social skills training. Impulse regulation was defined as raising hands before speaking. Effects on collateral behaviors (i.e., talking out of turn) were also assessed. A reversal design was used. Behavioral data collected by independent observers suggested that all subjects demonstrated positive changes in impulse regulation (i.e., an increase in the frequency with which subjects raised their hand before speaking). Likewise the treatment effects appeared to have produced positive effects on a behavior not directly targeted for intervention (i.e., talk outs). In general, behavioral changes were considered to be socially valid and the treatment agents viewed the cueing procedure very positively.
\end{abstract}

Attention Deficit Hyperactivity Disorder (ADHD), one of the most common childhood disruptive behavior disorders (DuPaul, 1991), is characterized by a consistent pattern of inattention and/or hyperactivity-impulsivity (American Psychiatric Association, 1994). Impulsivity is characterized by impatience and is often expressed in frequent interruptions of others, difficulty in delaying responses, and intruding on others (Rapport, 1994). Children with ADHD typically make comments out of turn, fail to listen to directions, initiate conversations at inappropriate times, blurt out answers before questions have been completed, grab objects from others, touch things inappropriately, and have difficulty waiting their turn (American Psychiatric Association, 1994).

Difficulties resulting from impulsivity are often exacerbated when children reach elementary school age and are faced with academic and social demands (DuPaul \& Stoner, 1994; Rapport, 1994). In elementary school settings, poor impulse regulation is often manifested in disruptive classroom behavior. Such behavior can negatively affect classroom instruction if the teacher must spend academic time controlling the child with ADHD rather than teaching. Children with ADHD who are also disruptive are likely to have academic difficulties if their impulse regulation substantially interferes with necessary learning behaviors, such as listening and following directions.

In addition to classroom interventions, the effectiveness of many treatment approaches for children with ADHD may be limited by poor impulse regulation. For example, social skills training, a common intervention that attempts to ameliorate the social deficits of children with ADHD, may have a diminished therapeutic effect if disruptive behavior significantly interferes with treatment delivery.

Impulsivity can be treated in a variety of ways. Behavioral therapies in particular have become a primary nonmedical treatment modality for the management of children with ADHD. Behaviorally based classroom interventions can help children with ADHD focus their attention and control their behavioral difficulties (Barkley, 1990; DuPaul \& Stoner, 1994; Fischer, Barkley, Edelbrock, \& Smallish, 1990; Nathan, 1992). Such treatments include operant conditioning and contingency-based reinforcement techniques (Abikoff \& Gittelman, 1984). When combined with interventions such as social skills training, behavioral management techniques may enhance the intervention's therapeutic impact.

This study sought to investigate the efficacy of a behavioral management technique aimed to facilitate impulse regulation in children with ADHD. The technique is referred to as a cueing procedure because it was 
designed to make target behaviors salient through multiple prompts or cues. The cueing procedure was aimed at increasing the frequency with which the subjects raised their hand before speaking, to help them devote attention to and improve impulse regulation. In addition, we believed that reinforcing subjects' hand raising behaviors may produce positive side effects on a related behavior (i.e., talking out of turn). Talking out of turn behaviors were not targeted directly; however, collateral effects on subjects' talking out behaviors were assessed.

For the purposes of this study, the facilitation of impulse regulation focused on increasing the frequency with which the children raised their hands before talking $(\mathrm{RH})$. Furthermore, effects on collateral behaviors were investigated by assessing talking out (TO) behaviors.

\section{METHOD}

\section{Subjects}

Four boys were selected out of a group of nine 8-year-olds involved in a summer social skills program. The subjects were selected because they (a) were similar in terms of type and severity of symptoms, (b) demonstrated identical target behaviors (i.e., low rates of hand raising and high rates of talking out), and (c) were enrolled for the entirety of an 8-week summer outpatient program.

All four subjects were diagnosed with ADHD and were receiving stimulant medication at the time of social skills training. The subjects' pattern of scores on the Child Behavior Checklist (CBCL) showed primary elevation on the externalizing dimension of behavioral dysfunction with factor scores elevated in the clinical range on aggressive behavior for each subject. Each of these children exhibited impulsivity and tended to (a) fail to raise their hand before speaking and (b) talk out of turn.

Only one of the four subjects was diagnosed exclusively with ADHD. The remaining subjects had dual diagnoses, including one of the following: depression, bipolar mood disorder, or a learning disability. It should be noted that comorbidity is high in children with ADHD (Barkley, 1990).

\section{Setting}

The cueing procedure was implemented at an 8-week outpatient treatment program for children with ADHD. The program included structured activities such as social skills training, academics, arts and crafts, and recreational activities. The cueing procedure was implemented and evaluated in the social skills component of the program. Nine 8-year-old boys participated in the social skills group that was led by two graduate students in Counseling Psychology. Social skills training consisted of loosely structured 50-minute sessions conducted daily. Skills taught during the sessions included listening, friendship making, and anger management with additional skills subsumed under these categories. In general, procedures used by the group leaders included discussion of each new skill followed by group interaction (e.g., role playing and topic related games).

Because the effects of the social skills training program were not of primary interest in this study, the social skill procedures were not standardized by the experimenters. Rather, this setting provided a context for implementing an intervention aimed specifically at increasing one form of subjects' impulse control (i.e., raising hand before speaking).

\section{Independent Variable}

The independent variable in this study was a cueing procedure implemented in the context of a loosely structured social skills program. Four methods of cueing were included in the intervention: visual reminders, goal evaluation, positive reinforcement, and constructive feedback (see Procedures section for more detail).

The primary components of the procedure are presented in Table 1. The target behavior (i.e., RH) was reinforced on a fixed interval schedule following short intervals (Houlihan \& Van Houten, 1989). The delivery of reinforcers was confined to a limited period of time to minimize distraction from the task (i.e., social skills training), because there is a tendency for children with ADHD to direct their attention away from the task and toward either the reinforcement itself or the person delivering the reinforcement (Douglas, Parry, Marton, \& Garson, 1976). Reinforcers were 
modified periodically to maintain the subjects' interest and decrease habituation to consequences (Barkley, 1990; Rapport, Tucker, DuPaul, Merlo, \& Stoner, 1986)

\section{TABLE 1}

Primary Components of Cueing Procedure

1. Children are given goal badges.

2. A timer is set and reset for 5 minute intervals.

3. A poster board containing the children's names is present in the front of the classroom.

4. A goal check occurs every 5 minutes.

5. Each child is evaluated individually.

6. Children are asked to evaluate their behavior.

7. Children are asked to evaluate each others' behavior.

8. Group leaders evaluate each child's behavior.

9. Goal attainment/improvement/no progress is determined by the group leaders.

10. Goal attainment is rewarded with a large sticker.

11. Goal improvement is rewarded with a small sticker.

12. Praise and encouragement are given for goal attainment and improvement.

\section{Dependent Measures}

The subjects' hand raising behaviors were evaluated via direct observations during social skills training. Likewise, behavioral generalization to nontargeted (i.e., collateral) behaviors was assessed by direct observation of talk outs. Behavioral data were collected during baseline and intervention conditions. Subjects' behaviors were also evaluated by the Conners Parent Rating Scale, which was completed prior to and following the 8-week treatment program.

Direct observations. Three independent observers conducted direct observations during social skills training. Operational definitions for the target and collateral behaviors were generated. Raising hand before speaking was counted if the child raised his hand prior to speaking and remained silent (did not emit words or noises) while his hand was raised. Talking out of turn was defined as the subject talking out when inappropriate (i.e., blurting out an answer or comment without being called on by the leader, making inappropriate noises that were unrelated to the discussion, or talking to a neighbor without permission).
Behavioral data were collected using a 2-minute partial interval sampling technique that allowed raters to simultaneously record both hand raising and talking out. Direct observations were conducted twice per week in 22-minute observation sessions across baseline and treatment conditions.

Observers were trained to mastery by the first author. Once observers attained at least $95 \%$ accuracy, they were assigned subjects to observe. Periodic meetings were conducted throughout the program to control for observer drift and to address questions or problems experienced by the observers. Twenty-five percent of the observations were conducted by two observers for the purpose of calculating interrater reliability. Cronbach's alpha was .98, demonstrating good reliability across observers.

Rating scales. Behavioral rating scales were used to obtain information regarding parent perceptions of behavior change. The Conners Parent Rating Scale (CPRS) (Conners, 1989) was used to measure child behaviors common to children with ADHD.

The CPRS (Conners, 1989) is a 48-item rating scale that assesses a breadth of behaviors common to ADHD. This scale has a mean of 50 and a standard deviation of 10. High scores indicate problem areas. Factor analysis revealed six factors on the CPRS (i.e., Conduct Problem, Learning Problem, Psychosomatic, Impulse-Hyperactivity, Anxiety, and Hyperactivity Index). The factors of greatest interest in this study were the Hyperactivity Index, Impulsive-Hyperactivity, and Conduct Problem. The scale was administered to parents prior to and following the 8-week summer treatment program.

\section{Procedure}

All children in the group were assigned a target or goal behavior. The goals were framed in terms of positive behaviors. All subjects in the study had the same goal for the duration of the program: Raise my hand before speaking. This was identified by the group leaders as priorities for target subjects. Other children in the group had goals such as look at the teacher, keep my hands to myself, or stay in the group. Four methods of cueing were used in this study. First, each child was given a personalized badge on which his target behavior was recorded. Badges were worn on the chil- 
dren's chests, similar to a name tag. These visual reminders served as the first method of cueing.

The second method of cueing involved frequent goal evaluation. Every 5 minutes, a timer sounded and a goal check was initiated. At that time, all children in the group (i.e., subjects and peers) were evaluated individually in three steps. First, the children were asked to self-evaluate (i.e., they were asked to report if they believed they had met their goal). It was believed that the process of self-evaluation would encourage selfmonitoring. Second, the children in the group were asked to evaluate whether each other met the goal. This process was believed to facilitate group support and cohesion. Finally, group leaders considered the selfand peer evaluations and made the final determination regarding goal attainment.

The third form of cueing involved positive reinforcement. If the child met his goal, he received praise and a large sticker that was publicly posted. The stickers were placed next to the child's name on a poster board located in the front of the classroom. If a child did not meet his goal but made progress toward it (as determined by the group leaders), a smaller sticker was publicly posted next to his name on the poster board. If the subject made no progress toward his goal, the fourth method of cueing, constructive feedback, was implemented. In this case, group leaders discussed methods by which subjects could alter their behaviors to attain their goal.

\section{Treatment Acceptability}

The treatment agents' (i.e., group leaders) acceptability of the cueing procedure was assessed following the 8-week treatment program. The Behavior Intervention Rating Scale (BIRS) (Von Brock \& Elliott, 1987) was completed by the group leaders at the end of the program. The BIRS is a 24-item scale with evidence of internal consistency (Von Brock \& Elliott, 1987) and factor validity (Elliott \& Von Brock Treuting, 1991). The factors on the scale include Acceptability (15 items), Effectiveness (6 items), and Time to Effectiveness (2 items). In this study, the
Acceptability factor was analyzed for purposes of assessing treatment acceptability.

\section{Social Validity}

Subjective evaluations of treatment effects were collected from rating scales and through informal interviews with the treatment agents. Specifically, four items of the BIRS Effectiveness factor were used to measure the treatment agents' perceptions of the effects of the cueing procedure on the subjects' overall social adjustment. ${ }^{1}$

\section{Treatment Integrity}

Treatment integrity of the cueing procedure was assessed using a structured checklist completed by an independent observer. The checklist was devised based on the treatment components in Table 1 . The 12 primary components of the intervention were listed and defined by the first author (see Table 1). Twenty-five percent of all treatment sessions were observed for purposes of assessing treatment integrity.

\section{Experimental Design}

A reversal $(\mathrm{ABAB})$ within-series design was used for all subjects. Behavioral data for RH and TO behaviors were collected across baseline and treatment conditions. Each experimental phase lasted for 2 weeks.

Changes in level between baseline and intervention conditions were analyzed. The percentage of overlapping data points was computed between baseline and the initial intervention phase, and the second baseline and second intervention phase for each behavior. Low overlap was suggested when $0 \%$ to $25 \%$ of treatment data overlapped with baseline data. Overlap of $26 \%$ to $49 \%$ overlap was considered moderate, and $50 \%$ or more overlap was considered high. Variation within conditions was computed using criteria suggested by Tawney and Gast (1984). Specifically, data within phases were considered stable if $80 \%$ to $90 \%$ of the data points of the condition were within a $20 \%$ range of the mean. 


\section{RESULTS}

Behavioral data for RH and TO behaviors are presented in Figures 1, 2,3 , and 4 . Average rates of behaviors for each subject and experimental phase are in Table 2.

Target behavior. As measured via direct observation procedures, Subjects 1 and 2 demonstrated change patterns from baseline to intervention conditions suggestive of experimental control for RH. Specifically, these subjects showed a substantial mean increase in the target behavior (RH) with the introduction of the cueing procedure, a return to baseline level during the second baseline, and an increase in frequency in the last intervention phase. Furthermore, change occurred immediately, and there were no overlapping data points for these subjects. Based on the criteria of Tawney and Gast (1984), the data remained variable across all experimental phases. The stability of the data is difficult to interpret given the level of the data and due to few data points in each phase.

Subject 3 showed the same general pattern; however, it was not until the second intervention phase that an increase in hand raising occurred. Subject 3 had high overlap between baseline and intervention conditions, although the percentage of overlap decreased by the second intervention phase. The data remained variable across all experimental phases.

Subject 4 appeared to have responded to the cueing procedure similar to Subjects 1 and 2; however, his data are difficult to interpret because he unexpectedly left the program prior to the last intervention phase. Subject 4 had a high degree of overlapping data points between the first baseline and intervention phases. The data in the first baseline and intervention phases remained relatively unstable and they returned to a low, relatively stable level during the second baseline phase.

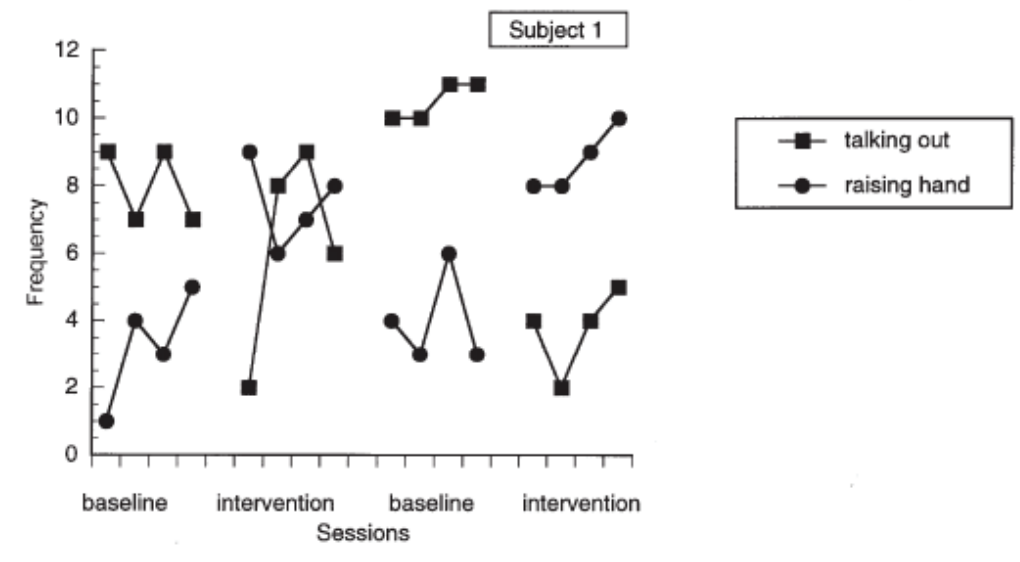

Figure 1. Direct observational data for hand raising (target) and talking out (collateral) behaviors for Subject 1.

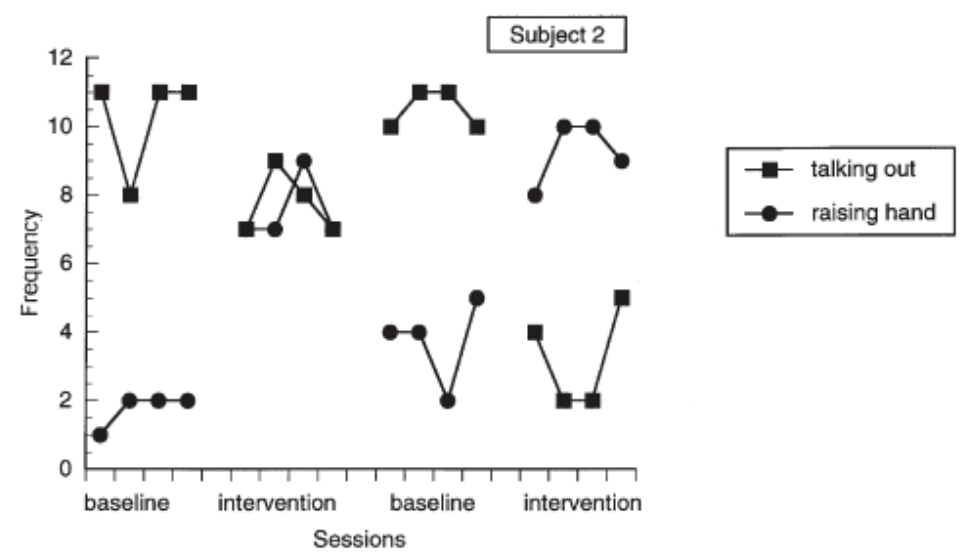

Figure 2. Direct observational data for hand raising (target) and talking out (collateral) behaviors for Subject 2. 


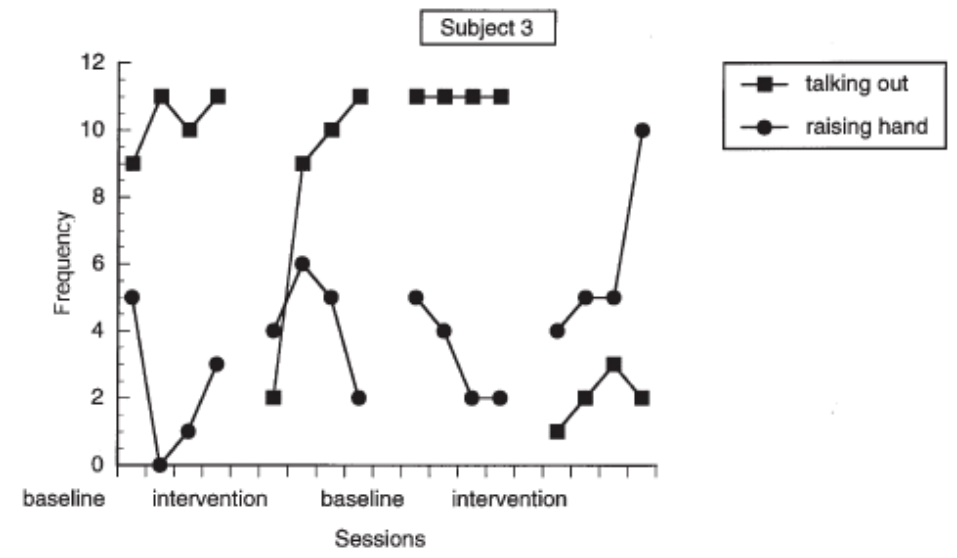

Figure 3. Direct observational data for hand raising (target) and talking out (collateral) behaviors for Subject 3.

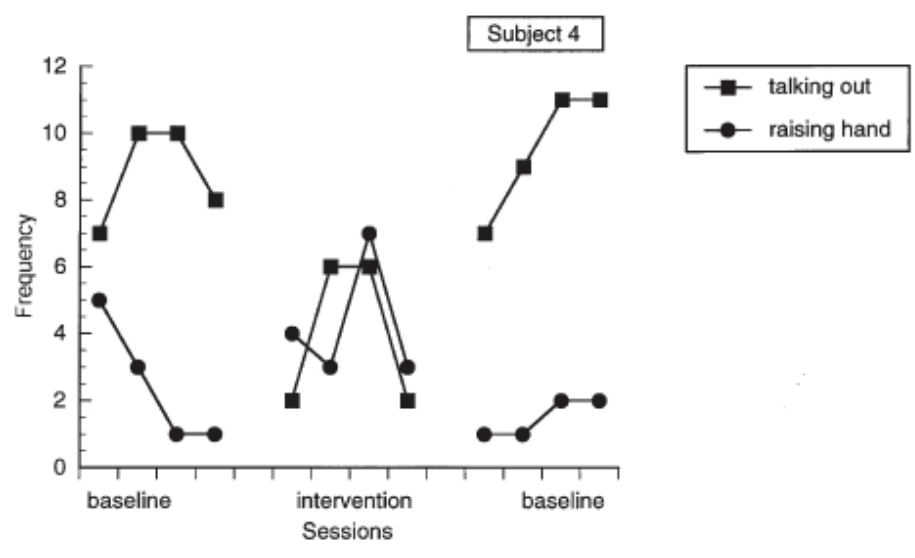

Figure 4. Direct observational data for hand raising (target) and talking out (collateral) behaviors for Subject 4.

\section{TABLE 2}

Average Number of Hand Raising and Talking Out Behaviors During Baseline and Intervention Phases

\begin{tabular}{lcccc}
\hline Subject & Baseline & Intervention & Baseline & Intervention \\
\hline Subject l & & & & \\
$\quad$ Raising hand & 3.25 & 7.50 & 4.00 & 8.75 \\
$\quad$ Talking out & 8.00 & 6.25 & 10.50 & 3.75 \\
Subject 3 & & & & \\
$\quad$ Raising hand & 2.25 & 4.25 & 3.25 & 6.00 \\
$\quad$ Talking out & 10.25 & 8.00 & 11.00 & 2.00 \\
Subject 2 & 1.75 & 7.50 & 3.75 & 9.25 \\
$\quad$ Raising hand & 10.25 & 7.75 & 10.50 & 3.25 \\
$\quad$ Talking out & & & & \\
Subject 4 & 2.50 & 4.25 & 1.5 & - \\
$\quad$ Raising hand & 8.75 & 4.00 & 9.5 & - \\
$\quad$ Talking out & & &
\end{tabular}

NOTE: Subject 4 left the treatment program before the final data collection period.

Collateral behaviors. A decrease in the mean level of talking out behaviors occurred with the cueing procedure; however, there seems to have been a delayed effect for most subjects. Subjects 1, 2, and 3 showed a substantial decrease in TO responses at the second intervention phase. In addition, the amount of overlap between baseline and intervention conditions diminished with the second intervention phase. Subject 4 is a possible exception in that he appears to have responded immediately to the first intervention phase with a decrease in TO responses and no overlapping data points. Unfortunately, data from the second intervention phase are not available for this subject.

Rating scale. The Conners Parent Questionnaire was administered preand posttreatment. Parents did not report improvements in their child's behavior on any of the factors of the Conners, suggesting the absence of perceived changes on behaviors not directly targeted by the cueing procedure. All subjects were in the clinical range pre-and post-test on the following factors: Hyperactivity Index, Impulsive-Hyperactivity, and Conduct Problem. 


\section{Treatment Acceptability}

The treatment agent's acceptability of the cueing procedure was assessed with the acceptability factor of the BIRS. The acceptability factor yielded a mean of 5.7 (highly agree). This suggests that the group leaders found the cueing procedure very acceptable.

\section{Social Validity}

Four items from the Effectiveness factor of the BIRS were analyzed to obtain a global rating from the group leaders regarding the degree to which the cueing procedure caused meaningful changes in the subjects' socialization. ${ }^{1}$ The mean, 4.0 (slightly agree), did not suggest meaningful changes in impulse control; however, anecdotal evidence from informal interviews with the treatment agents suggests that the cueing procedure improved the behavior of the subjects in the social skills setting, and consequently made the delivery of the social skills training easier.

\section{Treatment Integrity}

An independent observer conducted direct observations of $25 \%$ of the treatment sessions to assess whether treatment objectives were met by the treatment agents. Across sessions, 95\% of the essential components were observed, suggesting high fidelity of procedures by the group leaders.

\section{DISCUSSION}

\section{General Findings}

The cueing procedure appears to be an effective means of helping children with ADHD control their impulsive hand raising behaviors in a structured setting. All subjects demonstrated mean changes in the target behavior with the onset of the cueing procedure. Subject 3 did not respond to the cueing procedure until the second intervention

phase. This subject had a learning disability in conjunction with ADHD, which may have contributed to this apparent delayed effect.
It seems that increasing frequency with which subjects raised their hands before speaking actually improved behaviors that were related, but not directly targeted. Effects seem to generalize to talk out behaviors, as indicated by a decrease in mean levels.

Treatment effects for RH appear more substantial and immediate than effects for TO. This is understandable, given that only the RH behavior was targeted directly for intervention. TO was not directly treated, and it appears that effects on this collateral behavior occurred in a relatively delayed fashion.

It is important to note that the prioritization of appropriate target behaviors, particularly those that appear to functionally covary, can be an efficient means of addressing multiple behaviors within a relatively short period of time. The behaviors of interest in this study demonstrated an inverse relationship (one decreased in frequency as the other increased). The identification of the covariate relationship between responses resulted in an intervention that simultaneously modified two behaviors (albeit in a slightly delayed fashion), and obviated the need for sequential treatments aimed at changing only one behavior at a time (Parrish, Cataldo, Kolko, Neef, \& Egel, 1986).

The cueing procedure is properly described as a behavioral management technique rather than a form of therapy in and of itself. Accordingly, the procedure's impact seems limited to the specific behaviors targeted and does not directly address other deficits such as poor social skills and poor academic achievement, as well as the target behavior when the cueing procedure is not in effect. When used in conjunction with a therapeutic intervention (such as social skills training), however, the cueing procedure may enhance the intervention's impact. Unfortunately, no data on the effects of social skills training are available for this sample, and this speculation is left open to empirical test.

We suggest that our intervention was successful because it increased children's awareness of their target behaviors. When an ADHD child's goals are made salient as a result of a cueing procedure, he or she may be more likely to devote attention to improve target behaviors. The use of a cueing procedure with other target behaviors of children with ADHD also deserves empirical attention. 


\section{Contributions of the Study}

The present study contributes to the literature bases in interventions for children with ADHD, classroom interventions, and behavior modification techniques. Many interventions will be ineffective or will have limited effectiveness if the children's poor impulse regulation interferes with their attending to the treatment. This includes critical activities such as classroom learning, social skills training, and group therapy. This study combined the ideas of reinforcement with salience to improve impulse regulation, which may allow treatment agents to effectively deliver treatment and enable the children to attend to the treatment. However, this is an empirical question in need of further investigation.

The advantages of the cueing procedure are that it seems to work immediately for some and it can be taught easily and transferred from one setting to another. Group leaders are able to use it easily and with fidelity. Currently, however, it is unclear whether the procedure actually facilitates a therapeutic effect (e.g., helps children attend better to instruction or increase social skills learning).

\section{Limitations of the Study}

Although the cueing procedure represents a unique approach to controlling the poor impulse regulation of children with ADHD, several limitations of this study are apparent. These concern subjects and target behavior selection, dependent measures, short phases of experimental conditions, data analysis, and generalization issues.

Subject and target behavior selection. This study intended to test the effects of the cueing procedure on hand raising behaviors of children with ADHD. Reviews of subjects' records indicated that all subjects met the criteria for ADHD; however, no additional comprehensive diagnostic evaluations were conducted. Another limitation in the selection of subjects was that three of the four subjects had coexisting diagnostic conditions, although it should be noted that children with ADHD often have a dual diagnosis (Barkley, 1990). Finally, Subject 4 dropped out of the treatment program prior to the last intervention phase of the study.
Dependent measures. Several concerns are noted with the dependent measures used in this study. First, the direct observation method used to assess RH and TO was experimental, with no documentation of validity. It is believed that the procedures used to develop the observation scale enhanced the use of the procedures for this study (e.g., operational definitions of target behaviors were generated, observers were trained to mastery, and interrater reliability was adequate). Nevertheless, future research should investigate the psychometric properties of the observational method.

It is believed that controlling impulse regulation would allow the treatment agents to effectively deliver the social skills training and that it would enable the children to benefit more directly from the treatment. Informal interviews conducted with the treatment agents suggested that the cueing procedure made the delivery of the social skills training easier; however, no data were collected to determine if the cueing procedure actually led to better skill instruction or increased attention on the part of subjects.

An additional limitation of the present study was that social validity and treatment acceptability data were only collected from the treatment agents (i.e., group leaders). It would have been helpful to collect similar data from subjects themselves. Finally, rating scales (i.e., Conners) were completed by the parents and not the treatment agents. The lack of differences from pre-and posttreatment on this instrument may be explained by the fact that parents were not in a position to observe their children raising their hands in structured settings. The lack of reported differences may reflect a lack of generalization to other types of attentional, impulsive, or hyperactive behaviors, and not lack of treatment effects on the target behavior per se.

Experimental design. The short phases of the baseline and intervention conditions and consequent limited number of data points were a limitation of the study. Data collection was limited due to the length of the treatment program (8 weeks), which was out of the experimenters' control. Although the data appeared variable across many of the experimental conditions according to Tawney and Gast (1984), the instability of the data could be a function of the low data points. 
If the 8-week program were extended, impulse regulation and generalization effects might continue to improve. On the other hand, Ross and Ross (1982) have noted a frequent pattern of improvement and deterioration in classroom behavioral interventions. This pattern is believed to be influenced by inadequacies in treatment, habituation by the children to reinforcers, and/or poor teacher compliance. We believe that the latter outcomes would be less likely had the program been extended, as treatment integrity was high and reinforcers were modified as needed.

The 8-week program consisted of multiple components. A component analysis of the program was not included in the design, and the independent and combined effects of this program are unknown. Similarly, the influence of the social skills training program on the outcomes of the cueing procedure are unknown.

Data analysis. Subjects' responsiveness to the cueing procedure is difficult to ascertain. First, some variability within phases is apparent in the data. Second, ascending and descending trends are obvious for some subjects. Given these considerations, caution must be employed when drawing conclusions regarding the experimental control of the cueing procedure.

\section{Future Research Needs}

The purpose of the present study was to control poor impulse regulation in children with ADHD to enable a social skills intervention to take place. Although it appears that the cueing procedure successfully controlled impulsive behavior and made the delivery of the intervention easier for the group leaders, it is not clear whether the cueing procedure led to an increase in social skills learning. Several research needs can be identified to clarify the findings or further the procedure's usefulness.

First, it is important to resolve the limitations addressed above. Attention should be given to confirming the validity of the direct observational procedures. Direct measures of the group leaders' instructional behaviors, subjects' attention to tasks, and social skills should also be obtained to determine if controlling impulsivity actually leads to improved outcomes.
This study should be replicated with subjects that are exclusively ADHD, and a current psychological or diagnostic evaluation should be conducted routinely by researchers.

It may be beneficial to ascertain the most cost-effective interval for delivering reinforcers. In the current study, a goal check was conducted and reinforcers were delivered in 5-minute intervals. A longer interval may be less disruptive to the social skills training because there would be fewer interruptions. However, such an adaptation should be considered only if close monitoring assures that the cueing procedure's effectiveness is not diminished.

Although this study assessed the efficacy of the cueing procedure on subjects diagnosed with $\mathrm{ADHD}$, it is likely that other diagnostic populations may benefit from similar procedures. For example, it is possible that subjects with other conditions, such as Oppositional Defiant Disorder or Learning Disordered populations, may demonstrate positive treatment effects from cueing procedures.

Finally, the cueing procedure was implemented in one specific setting, social skills training. It is not known how it might affect behaviors in other settings, such as academic classrooms. In addition, the present study targeted a narrow subset of behaviors (i.e., raising hand before speaking). Furthermore, the subjects were all 8-year-old boys, most of whom had dual diagnoses. These factors, along with the small sample size, restrict the generalizability of the findings. Future research should attempt to determine if the results yielded in the present study can be generalized to other settings, behaviors, or diagnostic populations.

\section{NOTE}

1. The four items used to assess social validity were the following: the intervention produced lasting improvement in the child's behavior, the intervention improved the child's behavior to the point that it does not noticeably deviate from classmates', the child's behavior will remain at an improved level after the intervention is discontinued, and the intervention produced enough improvement in the child's behavior so that the behavior is no longer a problem. 


\section{REFERENCES}

Abikoff, H., \& Gittelman, R. (1984). Does behavior therapy normalize the classroom behavior of hyperactive children? Archives of General Psychiatry, 41, 449-454.

American Psychiatric Association. (1994). Diagnostic and statistical manual of mental disorders (4th ed.). Washington, DC: Author.

Barkley, R. A. (1990). Attention-deficit hyperactivity disorder: A handbook for diagnosis and treatment. New York: Guilford.

Conners, C. K. (1989). Conners rating scales manual. North Tonawanda, NY: MultiHealth Systems, Inc.

Douglas, V. I., Parry, P., Marton, P., \& Garson, C. (1976). Assessment of a cognitive training program for hyperactive children. Journal of Abnormal Child Psychology, 4, 389-410.

DuPaul, G. J. (1991). Parent and teacher ratings of ADHD symptoms: Psychometric properties in a community-based sample. Journal of Clinical Child Psychology, 20, 245-253.

DuPaul, G. J., \& Stoner, G. (1994). ADHD in the schools: Assessment and intervention strategies. New York: Guilford.

Elliott, S. N., \& Von Brock Treuting, M. (1991). The Behavior Intervention Rating Scale: Development and validation of a pretreatment acceptability and effectiveness measure. Journal of School Psychology, 29, 43-51.

Fischer, M., Barkley, R. A., Edelbrock, C. S., \& Smallish, L. (1990). The adolescent outcome of hyperactive children diagnosed by research criteria: 11. Academic, attentional, and neuropsychological status. Journal of Consulting and Clinical Psychology, 58, 580-588.

Houlihan, M., \& Van Houten, R. (1989). Behavioral treatment of hyperactivity: A review and overview. Education and Treatment of Children, 12, 265-275.

Nathan, W. A. (1992). Integrated multimodal therapy of children with attention-deficit hyperactivity disorder. Bulletin of the Menninger Clinic, 56, 283-312.

Parrish, J. M., Cataldo, M. F., Kolko, D. J., Neef, N. A., \& Egel, A. L. (1986). Experimental analysis of response covariation among compliant and inappropriate behaviors. Journal of Applied Behavior Analysis, 19, 241-254.

Rapport, M. D. (1994). Attention-deficit hyperactivity disorder. In V. B. Van Hasselt \& M. Hersen (Eds.), Advanced abnormal psychology (pp. 189-206). New York: Plenum.

Rapport, M. D., Tucker, S. B., DuPaul, G. J., Merlo, M., \& Stoner, G. (1986). Hyperactivity and frustration: The influence of control over and size of rewards in delaying gratification. Journal of Abnormal Child Psychology, 14, 191-204.

Ross, D. M., \& Ross, S. A. (1982). Hyperactivity: Current issues, research and theory (2nd ed.). New York: Wiley.

Tawney, J. W., \& Gast, D. L. (1984). Single subject research in special education. Columbus, OH: Merrill.

Von Brock, M. B., \& Elliott, S. N. (1987). Influence of treatment effectiveness information on the acceptability of classroom intervention. Journal of School Psychology, 25, 131-144.
Heidi D. Posavac received her doctorate in Counseling Psychology at the University of Utah. She is a staff psychologist and Senior Instructor of Psychiatry at Counseling and Mental Health Services at the University of Rochester. Her research interests include treatment outcome, program evaluation, and body image disturbance in women.

Susan M. Sheridan, Ph.D., is a professor of Educational Psychology (School Psychology). She received her doctorate in 1989 from the University of Wisconsin- Madison. She is vice president of Publications, Communications, and Convention Affairs for Division 16 (School Psychology) of the American Psychological Association, and associate editor for School Psychology Review. In 1993, she was awarded the Lightner Witmer Award by Division 16 (APA) for early career accomplishments. She has published several articles, chapters, and books, including The Tough Kid Social Skills Book (1995, Sopris-West) and Conjoint Behavioral Consultation: A Procedural Manual (1996, Plenum). Her primary research interests are in the areas of behavioral consultations and interventions, social skills training, and home-school partnership.

Steven S. Posavac received his doctorate in Social Psychology at the University of Utah. He is an assistant professor of marketing at the Simon Graduate School of Business Administration at the University of Rochester. His research interests include the role of attitudes in behavior and behavioral change, and consumer psychology. 